Proceedings

\title{
Oral health appointment in the context of COVID-19 pandemic: The contribute of infrared thermography
}

\author{
Miguel Pais Clemente ${ }^{1,6^{*}}$, André Moreira ${ }^{2}$, João Correia Pinto ${ }^{3}$ and José Manuel Amarante ${ }^{4,6}$ Joaquim Mendes 5,6 \\ 1 Departamento de Cirurgia e Fisiologia, Faculdade de Medicina, Universidade do Porto, Porto and Portugal; \\ mpaisclemente@med.up.pt \\ 2 Post Graduation in Oral Rehabilitation, Faculdade de Medicina Dentária, Universidade do Porto, Portugal; \\ mimd12064@fmd.up.pt \\ 3 Departamento de Cirurgia e Fisiologia, Faculdade de Medicina, Universidade do Porto, Portugal; \\ jcp1950@gmail.com \\ 4 Departamento de Cirurgia e Fisiologia, Faculdade de Medicina, Universidade do Porto, Porto, Portugal; \\ amarante@med.up.pt \\ 5 Faculdade de Engenharia, Universidade do Porto, Porto, Portugal; jgabriel@fe.up.pt \\ 6 INEGI, Labiomep, Universidade do Porto, Porto, Portugal; \\ * Correspondence: miguelpaisclemente@hotmail.com
}

Publisher's Note: MDPI stays neutral with regard to jurisdictional

claims in published maps and institutional affiliations.

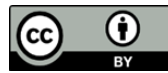

Copyright: (c) 2020 by the authors. Submitted for possible open access publication under the terms and conditions of the Creative Commons Attribution (CC BY) license (http://creativecommons.org/licenses /by/4.0/).

\begin{abstract}
Background: This research is focused on the oral health of a patient attending a dental appointment during the COVID-19 pandemic, where emergency treatments can have on infrared thermography (IRT), a technology that can be implemented as a screening method to quantify the temperature of the patient. IRT is an interesting bioengineering tool as it can provide more data to the clinician and even to the patient, who may not perceive the sublets fever sights from the begin; (2) Methods: Methods: This work analyzes anatomical landmarks on 98 frontal thermograms of the cranio-cervico-mandibular complex; (3) Results: The regions of interest for fever screening analyzed were the forehead, the inner canthus left eye, the inner canthus right eye with the mean temperature of $33.75{ }^{\circ} \mathrm{C}, 34.50{ }^{\circ} \mathrm{C}$ and $34.58{ }^{\circ} \mathrm{C}$ respectively. Significant statistically differences were observed comparing the temperature values of the forehead and the inner canthus. A greater temperature symmetry was found between the inner canthus of the left eye compared to the contralateral right eye; and (4) Conclusions: The authors intend to provide information that can stimulate a reflection on future directions for research and regulatory methods applied in dental medical sciences and how these measures can integrate the dental activity in a multidisciplinary model. In the future, as a consequence of this CoVID-19 disease, more efforts will be made to provide indispensable apparatus and devices that complement the already existing protection equipment. Within the area of bioengineering, the infrared thermography can be implemented as a protective additional measure in the clinical protocol during the attendance of a patient.
\end{abstract}

Keywords: oral health appointment; infrared thermography; fever screening; covid-19; infection control; body temperature; frontal thermograms; dentistry; personal protective equipment

\section{Introduction}

In December 2019, a novel coronavirus named SARS-CoV-2 emerged in Wuhan, China, and led to a rapidly spreading outbreak of coronavirus disease (COVID-19). By January 30th, COVID-19 was declared a Public Health Emergency (PHE) of international concern [1], by World Health Organization (WHO) [2].

Nevertheless, already in 2002, there was the recognition that the coronaviruses had established a potential cross species transmission originating a novel human coronavirus, the Severe Acute Respiratory Syndrome Coronavirus (SARS-CoV), that was first identified as an atypical pneumonia in isolated patients in Guangdong Province, China [3]. In China, two months after the emergence of SARS-CoV-2 the number of deaths re- 
lated to COVID-19 was already 2000 higher than that occurred during the 2003 SARS [4]. The similarities between SARS-CoV and SARS-CoV-2 are pertinent, since the whole genome of SARS-CoV-2 has $86 \%$ similarity with SARS-CoV [1]. Both viruses share high degrees of homology to SARS like coronaviruses isolated in bats, suggesting that bats are the probable origin of both, SARS-CoV and SARSCoV-2 [4]. Bats have been recognized as the natural reservoirs of a large variety of viruses. Special attention has been paid to bat as the two emerging coronaviruses which have caused unexpected human disease outbreaks in the 21st century, SARS-CoV [5] and Middle East Respiratory Syndrome Coronavirus (MERS-CoV) [6, 7].

The enzootic betacoronavirus MERS-CoV showed that humans can become infected within their communities, and transmission can then be amplified in the healthcare settings [10].

The SARS-coronavirus transmission appears to be through droplet spread, clearly placing dental personnel at risk, as they work in close proximity to their patients employing droplet and aerosol generating procedures [9], that can also be linked to other healthcare tasks such as intubation (71.4\%), airway suctioning (57\%), and sputum induction (28.6\%) [10].

This is one of the main issues that a dentist has to deal within an appointment during the COVID-19 phase, where special attention should be paid regarding the aerosol contamination. Despite the existing similarities on regulating and implementing measures to reduce the aerosols during the SARS-CoV and the SARS-CoV-2, one of the major differences is that in the first case the presence of fever may be a primary onset of the disease, while in the SARS-CoV-2 the patient can be infected and still asymptomatic with a high probability of transmit the COVID-19 disease. So, if a healthcare setting has a sensor device, like infrared thermography that can quantify the patient's temperature, this can be considered an added value on the screening of fever, providing more information for oral health care professionals.

The objective of this article is to describe the epidemiology, clinical features and the implementation of preventive measures such as infrared thermography during the COVID-19 pandemic on a dental appointment.

The study design of this research is divided in two parts. The first involves general considerations that should be taken in account within a dental appointment during the COVID-19 pandemic, with the handling of different personal protection equipment and the integration of infrared thermography. The second part concerns a retrospective revision of a database of infrared thermal images by measuring the body temperature of patients on two specific anatomical landmarks.

\section{Materials and Methods}

\subsection{Dental Appointment during the COVID-19 pandemic}

The dental appointment management and is based on four distinct moments: "The preparation for a dental appointment", "Dental set-up preparation", "Dental treatment" and "Post-treatment environmental cleaning".

\subsubsection{The preparation for a dental appointment}

During this phase, it is necessary to have the dental staff, namely the dental assistant or the administrative personal fully committed to the triage process, where a specific questionnaire should be answered by the patients that call to schedule an appointment or attend the clinic personally. The questions are targeted to screen patients with potential infection of 2019-nCoV before they could be led to the dental chair-side [11]. The Portuguese Dental Association (OMD) summarized some of the essential questions that should be asked [12]:

- "Have you travelled from a country that is considered as risk factor considering the transmission chain of COVID-19, on the last 14 days?" 
- "Do you have or had fever on the last 14 days?"

- "Do you have or had any respiratory problem, namely cough on the last 14 days?"

- "Did you have any type of unavoidable contact with a confirmed case of COVID-19?".

- "On the last 14 days, have you been in proximity with someone that presented acute respiratory problems?

- "Do you present symptoms, like fever, even if it was mild, fatigue sensation, cough, sneezes, conjunctivitis, diarrhea or rhinitis? (Any kind of symptoms that are compatible with COVID-19)".

\subsubsection{Dental set-up preparation}

In addition to the normal procedures of disinfection and sterilization, other measures of infection control precaution can be taken into practice, the oral health professional should reinforce the usual protective measures, since during this epidemic SARS-CoV-2 it is crucial to reduce the risk of contamination by respiratory droplets, therefore the personal protection equipment is fundamental for the skin and mucosa $[9$, 11, 13-16]. The protocol implemented by the authors regarding the dental appointment during the COVID-19 epidemic advocate the inclusion of infrared thermography. Furthermore, this research intends to use this technique as an additional equipment that until a certain extent can be a protective measure to the health professional, since the fever can be monitored with the thermal images without contact [17]. The dentist can thus check the temperature of the patient within the dental set-up, prior to the consultation (Figure 1).
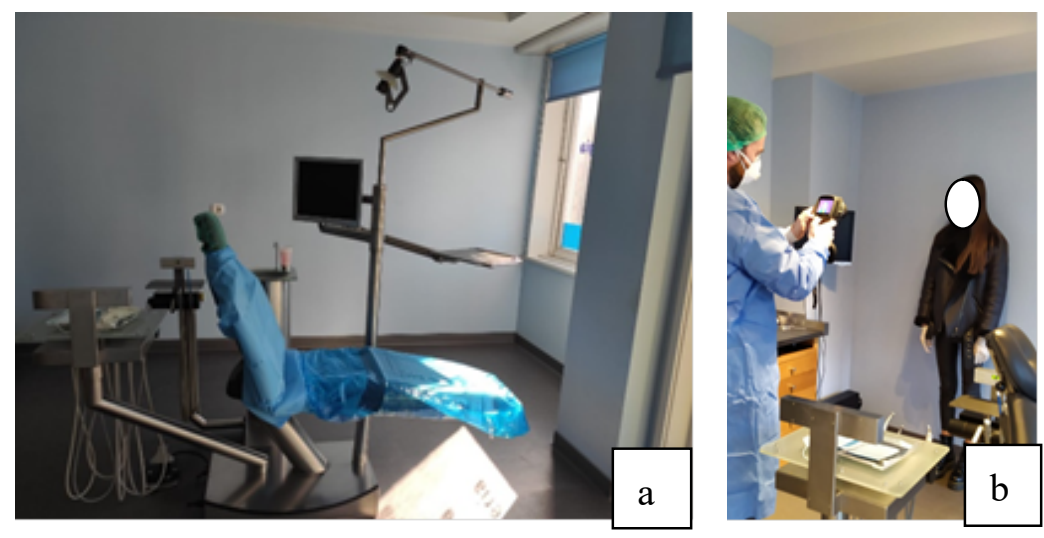

Figure 1. (a) Dental set-up with dental chair protection; (b) Dentist using thermal camera.

\subsection{Infrared thermal images capture}

The second part of this study was made concerning a retrospective revision of a database of infrared thermal images taken to patients who underwent the consultation of temporomandibular disorders of the C- HSJ, from April 2019 to January 2020. The sample size included 105 participants that signed an informed consent prior to the acquisition of the frontal thermograms. All participants were adults. This investigation is in accordance with the revised Helsinki Declaration (2013). Infrared frontal thermograms of the cranio-cervical-mandibular complex (CCMC) were performed using the FLIR i7 (Wilsonville, Oregon, USA) thermal camera at a distance of 1 meter from the patient, with the object of interest occupying $2 / 3$ of the image. The respective thermograms were analyzed on Flir Tools 6.4 software. The temperature values were measured on three distinct anatomical landmarks, the forehead, the inner canthus of the left and the right eyes. These three regions of interest (ROI) are of special interest for the detection of body temperature related to fever screening. The authors of this work intend to complement other re- 
searches that validate the ROI corresponding to the inner canthus of the eye as a reference point/area for fever monitoring.

A statistical analysis was done by obtaining the descriptive statistics, the Kolmogorov-Smirnov and Shapiro-Wilk tests were used to evaluate the normal distribution of the variables and later the Wilcoxon test were used to compare the variables.

\section{Results}

The sample consists of 105 frontal thermograms, from which 7 were discarded due to the incorrect angle between the face of the participant and the infrared camera. The remaining 98 were analyze using the forehead ROI (Fh) $(2-3 \mathrm{~cm}$ over the glabella), the inner canthus of the right eye (Re) and the inner canthus of the left eye (Le), Figures $4 \mathrm{a}$ ) and $b$.

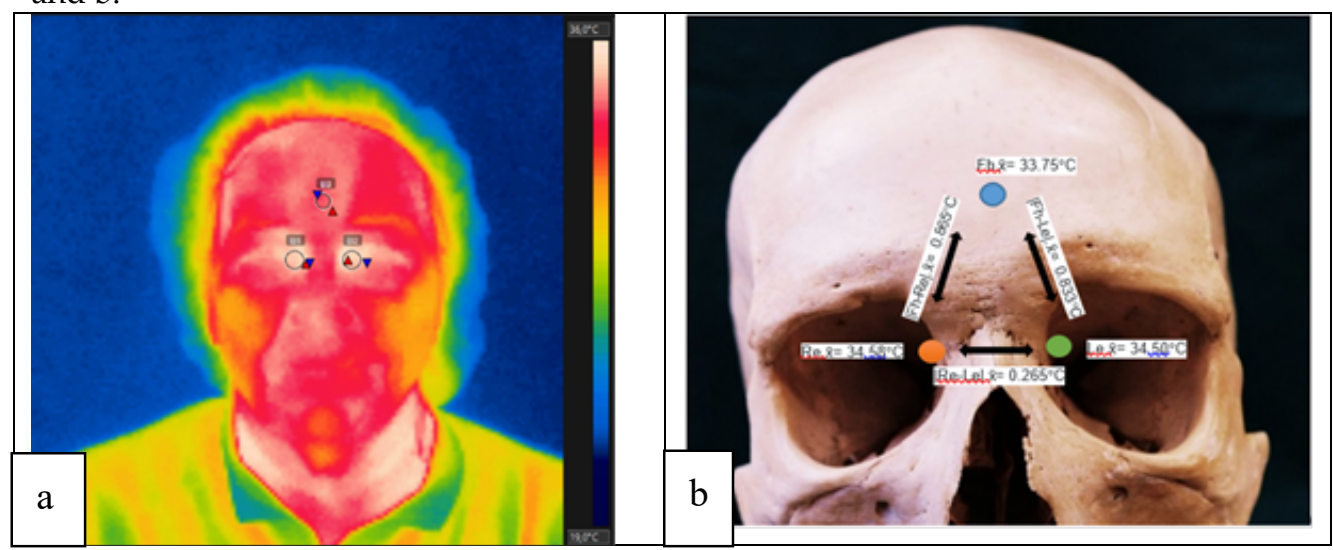

Figure 4. a) Frontal thermogram for screen fever. Analyses of the following regions of interest, the forehead (Fh), the inner canthus of the right eye (Re) and the inner canthus of the left eye (Le). b) Schematic illustration of the regions of interest - Blue circle represents the ROI for forehead (Fh) (2-3 cm over the glabella); Orange circle the inner canthus of the right eye (Re) and the green circle the inner canthus of the left eye (Le); $\bar{x}$ stands for mean value of the respective value; The double pointed arrow stands for the ROIs subtracted $(\Delta \mathrm{T})$.

It was also evaluated the absolute value of the thermal difference between ROIs: |Fh-Rel, |Fh-Lel and I Re-Lel that are shown in Table 1.

Table 1. Descriptive statistics for the studied variables.

\begin{tabular}{ccccc}
\hline Variables & Mean $\left({ }^{\circ} \mathbf{C}\right)$ & SD & min. & max. \\
\hline Fh & 33.75 & 1.046 & 31.30 & 35.90 \\
Re & 34.58 & 0.832 & 32.30 & 36.50 \\
Le & 34.50 & 0.870 & 31.70 & 36.30 \\
$\mid$ Fh-Re| & 0.865 & 0.514 & 0.00 & 2.20 \\
$\mid$ Fh-Le| & 0.833 & 0.510 & 0.00 & 2.00 \\
$\mid$ Re-Le| & 0.265 & 0.232 & 0.00 & 1.00 \\
\hline
\end{tabular}

The Kolmogorov-Smirnov and Shapiro-Wilk were tested for the 6 variables and only $\mid$ Fh-Re| followed a normal distribution $(p>0.05)$. Thus, to test the null hypothesis nonparametric tests were used.

-Null hypothesis 1: The temperature found for the Fh ROI is similar to the temperature at Re ROI;

-Null hypothesis 2: The temperature found for the Fh ROI is similar to the temperature at Le ROI;

-Null hypothesis 3: The temperature found for the Re ROI is similar to the temperature at Le ROI; 
-Null hypothesis 4: The $\Delta \mathrm{T}$ Fh-Le is similar to the $\Delta \mathrm{T}$ Fh-Re;

- Null hypothesis 5: The $\Delta \mathrm{T}$ Fh-Re is similar to the $\Delta \mathrm{T}$ Re-Le;

- Null hypothesis 6: The $\Delta \mathrm{T}$ Fh-Le is similar to the $\Delta \mathrm{T}$ Re-Le;

Taking into account the Wilcoxon tests the null hypothesis 1, 2, 3, 5 and 6 were rejected, once statistically differences were found $(\mathrm{p}<0.05)$. For the hypothesis 4 no statistically differences were found ( $p>0.05)$, Table 2.

Table 2. Wilcoxon tests.

\begin{tabular}{ccc}
\hline Hypothesis & Description & $p$ \\
\hline 1 & $\mathrm{Fh}\left({ }^{\circ} \mathrm{C}\right)$ vs $\operatorname{Re}\left({ }^{\circ} \mathrm{C}\right)$ & 0.000 \\
2 & $\operatorname{Fh}\left({ }^{\circ} \mathrm{C}\right)$ vs Le $\left({ }^{\circ} \mathrm{C}\right)$ & 0.000 \\
3 & $\operatorname{Re}\left({ }^{\circ} \mathrm{C}\right)$ vs Le $\left({ }^{\circ} \mathrm{C}\right)$ & 0.020 \\
4 & $\Delta \mathrm{T}$ Fh-Le vs $\Delta \mathrm{T}$ Fh-Re & 0.068 \\
5 & $\Delta \mathrm{T}$ Fh-Re vs $\Delta \mathrm{T}$ Re-Le & 0.000 \\
6 & $\Delta \mathrm{T}$ Fh-Le vs $\Delta \mathrm{T}$ Re-Le & 0.000 \\
\hline
\end{tabular}

\section{Discussion}

Now that it is possible to understand the chronology of events that occurred with the initial infections of the 2019-nCoV, apparently linked to contact with animals in wet markets, in the City of Wuhan, China, several cases were documented with atypical pneumonia. Ralph et al. reports that initially, four cases noted presented fever (greater than $38^{\circ} \mathrm{C}$ ), general weakness, dry cough, and shortness of breath [18]. SARS-CoV-2 has the similarity to the SARS-CoV and the MERS-CoV epidemics the occurrence of travel-related cases [18]. Since the $13^{\text {th }}$ of January until the 20th of January different cases of 2019-Cov were reported in Thailand (twice), Japan, Nepal and South Korea and all of them had in common the fact that subjects have been recently in Wuhan. After these occurrences, several airports in Asia and North America have set up thermal screening at airports to detect possible 2019-nCoV infected individuals.

Chairman Childs presented valid and very important information throughout a chapter entitled "body temperature and clinical thermometry" highlighting that non-invasive, non-contact skin thermometry is ideally suited and provides an easier way for regular monitoring of body temperature [19]. The most commonly used non-contact method in clinical practice is the infrared thermography [19]. In fever screening, it is widely acknowledged that the internal core organs are the hottest regions of the human body [19]. Chairman Childs explains in perfection the true representation of core temperature, since it can be interpreted as the "thermoregulatory center" itself, the hypothalamus, a small area of gray matter, deep within the center of the cerebral hemispheres (the diencephalon), that represents a true measure to be considered as core temperature. Brain tissue is shielded from the environment due to its location within the skull and, along with the constant bathing of the brain by incoming arterial blood. One of the key targets of thermography is the forehead, but other sites may be more reliable. One area under investigation is a small region on the face where temperature is highest; the skin between the medial aspect of the orbit and the nose, the inner canthus, where a rich network of vessels supplies this small area, originated by the ophthalmic artery (supratrochlear branch) [20]. Actually the writings of Chairman Childs are in the same line of a previous publication of Francis Ring and Kurt Ammer on "Infrared thermal imaging in medicine", these authors mentioned that a close up image of the upper face, where a minimum of 9 pixels can be located in each corner of the eye the inner canthus that will provide a true indication of the presence or absence of fever [21].

The results of our study are in total accordance with the previous investigations of Francis Ring and Kurt Ammer, and Chairman Childs related to the inner canthus being the ideal ROI for fever screening at a dental appointment. The results of the current investigation with the analyze of the 98 thermograms show that it was statistical significant 
measuring the body temperature of the inner canthus ROI comparing to the forehead ROI. The regions of interest for fever screening were the forehead, the inner canthus left eye, the inner canthus right eye with the mean temperature of $33.75,34.50$ and $34.58^{\circ} \mathrm{C}$ respectively. A greater symmetry of temperature was found between the inner canthus of the left eye and the inner canthus of the right eye. Another important finding is that temperature has a minimal difference of $0.2^{\circ} \mathrm{C}$ when comparing the values of the inner canthus of the left eye with the temperature values of the inner canthus of the right eye. Thus, the inner canthus is a reproducible and valid option for monitoring the body temperature when a healthcare provider pretends to screen the fever of a patient attending a dental appointment. To our knowledge, the inner canthus is the ROI that represents the correct anatomical area for the registration of the most accurate temperature of a person corresponding to the core temperature.

This data is important for healthcare workers that use thermal cameras for fever screening, to drop out the use of the forehead as a ROI, since it is not accurate and does not correspond to the highest temperature value that is possible to achieve at a frontal thermogram of the cranio-cervico-mandibular complex.

\section{Conclusions}

The region of interest for fever screening analyzed on the frontal thermograms of the cranio-cervico-mandibular complex indicate that the inner canthus of the eye is the region of interest that should be taken in consideration for fever screening. Infrared thermography can be an additional tool to use in a dental appointment for fever screen since this technique is non-invasive, reproducible, does not emit radiation, allows to maintain a security distance from the patient pandemic while assessing body temperature, nevertheless it is fundamental to understand the limitations and use a rigorous protocol.

Dentistry has a responsibility towards society, towards science and towards different types of pathologies that have appeared along these years. A multidisciplinary approach for SARS-CoV-2 is fundamental, where a specific COVID-19 task force should take special attention to oral health in order to present valid solutions on the study and diagnoses of this pandemic event, including the use of infrared thermography.

Author Contributions: Conceptualization, M.P.C.; methodology M.P.C.; software, J.M.; validation, J.M., J.C.P. and J.M.A.; investigation, M.P.C.; resources, J.M.; data curation, M.P.C.; writing-original draft preparation, M.P.C.; writing - review and editing, J.M., A.M.; visualization, M.P.C; supervision, J.M., J.C.P. and J.M.A; funding acquisition, J.M. All authors have read and agreed to the published version of the manuscript.

Funding: The authors would like to acknowledge the support of the project "Simplified - Easy Tooth Abutment", ref. POCI-01-0247-FEDER-017982, co-founded by Fundo Europeu de Desenvolvimento Regional (FEDER), through Programa Operacional Competitividade e Internacionalização (POCI) and the support of INEGI - Institute of Science and Innovation in Mechanical and Industrial Engineering, through the project LAETA - UIDB/50022/2020 and UIDP/50022/202.

Acknowledgments: The authors would like to the patients of Centro Hospitalar de São João, for their collaboration.

Conflicts of Interest: The authors declare no conflict of interest.

\section{References}

1. Chan JF-W, Kok K-H, Zhu Z, Chu H, To KK-W, Yuan S, et al. Genomic characterization of the 2019 novel human-pathogenic coronavirus isolated from a patient with atypical pneumonia after visiting Wuhan. Emerging Microbes \& Infections.

2020;9(1):221-36.

2. World Health Organization. Coronavirus disease (COVID-19) outbreak. 2020. Available at:

https://www.who.int/emergencies/ diseases/novel-coronavirus-2019 [last accessed February 2020].

3. Bolles M, Donaldson E, Baric R. SARS-CoV and emergent coronaviruses: viral determinants of interspecies transmission. Current Opinion in Virology. 2011;1(6):624-34. 
4. Wilder-Smith A, Chiew CJ, Lee VJ. Can we contain the COVID-19 outbreak with the same measures as for SARS? The Lancet Infectious Diseases.

5. $\quad \mathrm{Hu}$ B, Ge X, Wang L-F, Shi Z. Bat origin of human coronaviruses. Virology Journal. 2015;12.

6. Raj VS, Osterhaus ADME, Fouchier RAM, Hagmans BL. MERS: emergence of a novel human coronavirus. Current Opinion in Virology. 2014;5:58-62.

7. Al-Osail AM, Al-Wazzah MJ. The history and epidemiology of Middle East respiratory syndrome corona virus. Multidisciplinary Respiratory Medicine. 2017;12(1):20.

8. Al-Tawfiq JA, Memish ZA. Middle East respiratory syndrome coronavirus: transmission and phylogenetic evolution. Trends in microbiology. 2014;22(10):573-9.

9. Li RW, Leung KW, Sun FC, Samaranayake LP. Severe acute respiratory syndrome (SARS) and the GDP. Part II: implications for GDPs. British dental journal. 2004;197(3):130-4.

10. Memish ZA, Zumla AI, Assiri A. Middle East respiratory syndrome coronavirus infections in health care workers. The New England journal of medicine. 2013;369(9):884-6.

11. Peng X, Xu X, Li Y, Cheng L, Zhou X, Ren B. Transmission routes of 2019-nCoV and controls in dental practice. International journal of oral science. 2020;12(1):9.

12. OMD. Recomendações para situações de urgência e inadiáveis - Ordem dos Médicos Dentistas 2020 [Available from: https://www.omd.pt/covid-19/urgencias/?fbclid=IwAR0_cHUYYzWgpf5Hz30t_H89L02Vb9TLpVLRvalRh6F79clbc27DW0MfjfQ.

13. Yan Y, Chen H, Chen L, Cheng B, Diao P, Dong L, et al. Consensus of Chinese experts on protection of skin and mucous membrane barrier for health-care workers fighting against coronavirus disease 2019. Dermatologic therapy. 2020:e13310.

14. Meng L, Hua F, Bian Z. Coronavirus Disease 2019 (COVID-19): Emerging and Future Challenges for Dental and Oral Medicine. Journal of dental research. 2020:22034520914246.

15. Freitas MdGGd. Prevenção e Controlo de Infeção por SARS-CoV-2 (COVID-19): Equipamentos de Proteção Individual (EPI). In: Saúde Dgd, editor. 2020.

16. CDCP. Dental Settings - Interim Infection Prevention and Control Guidance for Dental Settings During the COVID-19 Response - Centers for Disease Control and Prevention 2020 [Available from:

https://www.cdc.gov/coronavirus/2019-ncov/hcp/dental-settings.html.

17. Ring EFJ, Jung A, J.Zuber. Infrared Imaging. A casebook in clinical medicine2015.

18. Ralph R, Lew J, Zeng T, Francis M, Xue B, Roux M, et al. 2019-nCoV (Wuhan virus), a novel Coronavirus: human-to-human transmission, travel-related cases, and vaccine readiness. Journal of infection in developing countries. 2020;14(1):3-17.

19. Childs C. Chapter 29 - Body temperature and clinical thermometry. In: Romanovsky AA, editor. Handbook of Clinical Neurology. 157: Elsevier; 2018. p. 467-82.

20. Erdogmus S, Govsa F. Arterial Features of Inner Canthus Region: Confirming the Safety for the Flap Design. Journal of Craniofacial Surgery. 2006;17(5):864-8.

21. Ring EF, Ammer K. Infrared thermal imaging in medicine. Physiological measurement. 2012;33(3):R33-46. 